Please do not remove this page

RMIT

UNIVERSITY

\title{
The acoustic radiation impedance of a rectangular panel
}

Davy, John; Larner, David; Wareing, Robin; Pearse, John

https://researchrepository.rmit.edu.au/esploro/outputs/9921862705201341/filesAndLinks?institution=61RMIT_INST\&index=null

Davy, J., Larner, D., Wareing, R., \& Pearse, J. (2015). The acoustic radiation impedance of a rectangular panel. Building and Environment: The International Journal of Building Science and Its Applications, 92, 743-755. https://doi.org/10.1016/j.buildenv.2015.05.042

Document Version: Accepted Manuscript

Published Version: https://doi.org/10.1016/j.buildenv.2015.05.042

Repository homepage: https://researchrepository.rmit.edu.au

(C) 2015 Elsevier Ltd. All rights reserved.

Downloaded On 2023/04/26 23:38:43 +1000

Please do not remove this page 
Thank you for downloading this document from the RMIT Research Repository.

The RMIT Research Repository is an open access database showcasing the research outputs of RMIT University researchers.

RMIT Research Repository: http://researchbank.rmit.edu.au/

\section{Citation:}

Davy, J, Larner, D, Wareing, R and Pearse, J 2015, 'The acoustic radiation impedance of a rectangular panel', Building and Environment: the international journal of building science and its applications, vol. 92, pp. 743-755.

See this record in the RMIT Research Repository at:

https://researchbank.rmit.edu.au/view/rmit:32181

Version: Accepted Manuscript

Copyright Statement: (C) 2015 Elsevier Ltd.Creative Commons AttributionNonCommercial-NoDerivatives 4.0 International License.

Link to Published Version:

http://dx.doi.org/10.1016/j.buildenv.2015.05.042 


\section{The Acoustic Radiation Impedance of a Rectangular Panel}

John Laurence Davy ${ }^{\mathrm{a}}$, David James Larner ${ }^{\mathrm{a}}$

Robin R. Wareing ${ }^{\mathrm{b}}$, John R. Pearse ${ }^{\mathrm{b}}$

${ }^{a}$ School of Applied Sciences, Royal Melbourne Institute of Technology (RMIT) University, GPO Box 2476, Melbourne, Victoria 3001, Australia, tel. +61422171812, fax +61399255290, john.davy@.rmit.edu.au, david.larner@rmit.edu.au

${ }^{\mathrm{b}}$ University of Canterbury, Mechanical Engineering, Private Bag 4800, Christchurch 8140, New Zealand, robin.wareing@pg.canterbury.ac.nz, john.pearse@canterbury.ac.nz

Corresponding author: John Laurence Davy

\section{Abstract}

This paper extends the definition of the one sided radiation impedance of a panel mounted in an infinite rigid baffle which was previously used by the authors so that it can be applied to all transverse velocity wave types on the panel rather than just to the possibly forced travelling plane transverse velocity waves considered previously by the authors. For the case of travelling plane waves on a rectangular panel with anechoic edge conditions, and for the case of standing waves on a rectangular panel with simply supported edge conditions, the equations resulting from one of the standard reductions from quadruple to double integrals are given. These double integral equations can be reduced to single integral equations, but the versions of these equations given in the literature did not always converge when used with adaptive integral routines and were sometimes slower than the double integral versions. This is because the terms in the integrands in the existing equations have singularities. Although these singularities cancel, they caused problems for the adaptive integral routines. This paper rewrites these equations in a form which removes the singularities and enables the integrals in these equations to be evaluated with adaptive integral routines. Approximate equations for the azimuthally averaged one sided radiation impedance of a rectangular panel mounted in an infinite baffle are given for all the cases considered in this paper and the values produced by these equations are compared with numerical calculations.

Key Words: Acoustic radiation impedance; Acoustic radiation efficiency

\section{Introduction}

The acoustical radiation impedance of one side of a finite rectangular panel mounted in an infinite rigid baffle is of importance for the prediction of sound insulation [1-5], sound absorption [1, 68], sound directivity [9] and sound scattering. It occurs naturally when variational techniques are used to solve these phenomena [1, 2, 7, 8]. The normalized real part of the acoustical radiation impedance of one side of a finite rectangular panel mounted in an infinite rigid baffle is also the panel's one sided acoustic radiation efficiency.

The authors [10-12] have recently defined the radiation impedance of a plane panel mounted in an infinite plane baffle as the average of the specific acoustic impedance over the surface of the rectangular panel when a possibly forced plane transverse velocity wave is propagating on the surface of the rectangular panel. It was assumed that the edges of the panel were anechoic. This is the appropriate assumption for a forced wave, because after the forced wave is reflected at the edges of the panel, it propagates with the free wave number of the panel rather than with the forced wave number and hence has a different radiation impedance unless the incident wave was also freely propagating.

This definition works because the possibly forced plane wave has the same root mean square (rms) transverse velocity over time at all points of the panel. When the radiation impedance of 
other wave types on the panel, such as standing waves, is considered, this definition breaks down because the rms transverse velocity over time will possibly differ over the panel and may be zero at some points. Where the rms transverse velocity over time is zero, the specific acoustic impedance will be infinite and its average over the panel may not be finite. This paper gives a definition of the radiation impedance of a transverse wave on a panel which gives the same result for a travelling plane wave as the definition previously used by the authors.

The definition of radiation impedance involves a quadruple integral. For a rectangular panel with a travelling plane wave or for a mode of a simply supported panel, this quadruple integral can be reduced to a double integral using a standard technique [1, 13-15]. In both these cases this double integral can be reduced to a much more complicated single integral. However when the single integral equations for real part of the impedance for the travelling plane case [15] were evaluated using adaptive integral routines, the integral did not converge when the wave number of the travelling transverse plane velocity wave was equal to the wave number in the fluid medium into which the panel was radiating. Also, at low frequencies, the single integral evaluation was slower than the double integral evaluation. These problems are due to singularities in terms of the integrand. Although the singularities do cancel out each other, they do cause problems for the adaptive integral routines. Singularities also appear in the single integral formulae for the real part of the impedance for the simply supported mode case [13]. This paper rewrites these integrands in a form that removes the singularities, so that the adaptive integral routines work correctly and effectively. This paper also derives the single integral formulae for the imaginary part of the impedance for both the travelling wave case and the simply supported mode case. Most previous papers only treat the travelling plane wave case or the simply supported mode case. This paper gives a uniform treatment of both cases.

Even with one less level of integration, the numerical evaluation can be fairly time consuming, especially as the products of the wave number of the transverse velocity wave in the panel and the wave number of the sound in air with the half side lengths of the panel become large. Thus, this paper also gives approximate formulae and compares their output with the numerical calculations for the azimuthally averaged one sided impedance of a square panel mounted in an infinite rigid baffle. Approximate formulae are also given for the case when the waves in the panel are excited by a diffuse sound field which is incident on one side of the panel.

When a panel is actually excited, there are usually at least two types of transverse vibrational fields excited in the panel. One is a freely propagating resonant field and the other is a forced non-resonant field or a near field. Equations are given for calculating the impedance of a panel in an infinite baffle which is excited by an incident diffuse sound field, by transverse point forces or by transverse line forces.

This paper also examines the difference in radiation impedance between different types of waves. At first sight, it is surprising that there are differences in some cases between the radiation impedances of travelling plane waves and simply supported modes on a rectangular isotropic panel, because the simply supported modes can be expressed as a sum of travelling waves. The reason for the differences are that one wave on the panel can alter the impedance experienced by another wave. This also applies to the real part of the normalized radiation impedance of different modes on a panel, but Xie et al. [16] have shown that these modal interactions cancel out when the position of the transverse excitation point is averaged over the surface of the panel. The authors suspect that a similar cancellation of the interactions between different travelling waves or simply supported modes occurs when azimuthal averaging or incident diffuse field averaging is used. This is because the results of such averaged results have proved useful in making acoustical predictions. Such cancellation does not always occur when the travelling plane waves are summed to form a mode because the relative phase of the travelling plane waves is fixed by the boundary conditions of the panel. Hence these differences in impedance survive the azimuthal averaging. 


\section{Definition of radiation impedance}

In this paper, the sinusoidal variation with time is assumed to be proportional to exp $(j \omega t)$, where $\omega$ is the angular frequency, $t$ is the time, $j$ is the square root of -1 . It should be noted that the assumption of $\exp (-j \omega t)$ for the sinusoidal variation with time gives the opposite sign for the imaginary part of the impedance. The impedances in this paper are normalized by dividing by the characteristic impedance of the fluid medium $Z_{c}$, which is the product of the ambient density of the fluid medium $\rho_{0}$ and the speed of sound in the fluid medium $c$. Note that root mean square (rms) amplitudes rather than peak amplitudes are used in this paper.

Consider a plane surface area $S$ whose area is also denoted by $S$, mounted in an infinite rigid plane baffle in the $x-y$ plane $z=0$, in which a two dimensional transverse velocity wave is propagating. The rms transverse velocity of the wave over the surface area of the panel in the positive $\mathrm{z}$-axis direction is $u\left(\mathbf{r}_{0}\right)$ where $\mathbf{r}_{0}=\left(x_{0}, y_{0}, z_{0}\right)$ is the position on the panel. The sound pressure in the fluid medium on the positive $z$ side of the baffle at position $\mathbf{r}_{1}=\left(x_{1}, y_{1}, z_{1}\right)$ is given by the Rayleigh integral (See Eq. (2.4) of [17])

$$
p\left(\mathbf{r}_{1}\right)=j k Z_{c} \iint_{S} u\left(\mathbf{r}_{0}\right) g_{\omega}\left(\mathbf{r}_{1}, \mathbf{r}_{0}\right) d \mathbf{r}_{0}
$$

where $g_{\omega}$ is the Green's function for a point source on an infinite rigid baffle which is given by

$$
g_{\omega}\left(\mathbf{r}_{1}, \mathbf{r}_{0}\right)=\exp (-j k r) /(2 \pi r)
$$

where

$$
r=\left|\mathbf{r}_{1}-\mathbf{r}_{0}\right|=\sqrt{\left(x_{1}-x_{0}\right)^{2}+\left(y_{1}-y_{0}\right)^{2}+\left(z_{1}-z_{0}\right)^{2}}
$$

and $k$ is the wave number in the fluid medium into which the wave is radiating on the positive $z$ side of the baffle.

The sound power $W$ radiated by one side of the panel is

$$
W=\operatorname{Re}\left[\iint_{S} p\left(\mathbf{r}_{1}\right) u^{*}\left(\mathbf{r}_{1}\right) d \mathbf{r}_{1}\right]=\operatorname{Re}\left[j k Z_{c} \iint_{S} \iint_{S} u\left(\mathbf{r}_{0}\right) u^{*}\left(\mathbf{r}_{1}\right) g_{\omega}\left(\mathbf{r}_{1}, \mathbf{r}_{0}\right) d \mathbf{r}_{0} d \mathbf{r}_{1}\right] .
$$

It is desirable to be able to write the sound power $W$ radiated by one side of the panel as

$$
W=\operatorname{Re}\left[z Z_{c} S\left\langle u^{2}\right\rangle\right]
$$

where

$$
\left\langle u^{2}\right\rangle=\iint_{S}\left|u\left(\mathbf{r}_{0}\right)\right|^{2} d \mathbf{r}_{0} / S=\iint_{S} u\left(\mathbf{r}_{0}\right) u^{*}\left(\mathbf{r}_{0}\right) d \mathbf{r}_{0} / S
$$

is the mean square transverse velocity of the plane surface area $S$. Hence it is convenient to define the normalized radiation impedance $z$ of a wave on the surface $S$ as

$$
z=j k \iiint_{S} \int_{S} g_{\omega}\left(\mathbf{r}_{1}, \mathbf{r}_{0}\right) u\left(\mathbf{r}_{0}\right) u^{*}\left(\mathbf{r}_{1}\right) d \mathbf{r}_{0} d \mathbf{r}_{1} /\left(S\left\langle u^{2}\right\rangle\right)
$$

If the transverse velocity of the plane wave on the surface $S$ in the positive $z$-axis direction is

$$
u\left(\mathbf{r}_{0}\right)=u_{0} \exp \left(-j \mathbf{k}_{b} \cdot \mathbf{r}_{0}\right)
$$

where $\mathbf{k}_{b}=\left(k_{x}, k_{y}, 0\right)$ is the wave number vector of the wave and $u_{0}$ is the complex amplitude of the wave, then

$$
\left\langle u^{2}\right\rangle=|u|_{0}^{2}
$$

and

$$
z=j k^{2} \iint_{S} \iint_{S} \exp \left[j \mathbf{k}_{b} \cdot\left(\mathbf{r}_{1}-\mathbf{r}_{0}\right)\right][\exp (-j k r) /(k r)] d \mathbf{r}_{0} d \mathbf{r}_{1} /(2 \pi S)
$$


This agrees with equation (10) of [11] which was derived using the authors' previous definition.

If the transverse velocity of the standing wave on the surface $S$ in the positive $z$-axis direction is

$$
u\left(\mathbf{r}_{0}\right)=u_{0} \sin \left(k_{x} x_{0}\right) \sin \left(k_{y} y_{0}\right)
$$

then

$$
\left\langle u^{2}\right\rangle=|u|_{0}^{2} / 4
$$

where it has been assumed that $u\left(\mathbf{r}_{0}\right)$ is zero on all four edges of the panel. In this case

$$
z=2 j k \iint_{S} \iint_{S} \sin \left(k_{x} x_{0}\right) \sin \left(k_{y} y_{0}\right) \sin \left(k_{x} x_{1}\right) \sin \left(k_{y} y_{1}\right)[\exp (-j k r) /(k r)] d x_{0} d y_{0} d x_{1} d y_{1} /(\pi S) .
$$

The real part of this equation agrees with equation (2.5) of [13] for the radiation efficiency which is the real part of the normalized radiation impedance.

\section{Reduction to double integral}

If $S$ is the rectangle

$$
|x| \leq a,|y| \leq b, z=0,
$$

then the quadruple integrals in equations (10) and (14) can be reduced to double integrals using the methods of Appendix A of [14], Appendix 12.A of [1], [15] and [13]. Equations (10) and (13) become

$$
\begin{gathered}
z=j k^{2} \int_{0}^{2 a} \int_{0}^{2 b}(2 a-\kappa) \cos \left(k_{x} \kappa\right)(2 b-\tau) \cos \left(k_{y} \tau\right) \\
{\left[\exp \left(-j k \sqrt{\kappa^{2}+\tau^{2}}\right) /\left(k \sqrt{\kappa^{2}+\tau^{2}}\right)\right] d \kappa d \tau /(2 \pi a b)}
\end{gathered}
$$

and

$$
\begin{gathered}
z=j k^{2} \int_{0}^{2 a} \int_{0}^{2 b}\left[(2 a-\kappa) \cos \left(k_{x} \kappa\right)+\kappa \mathrm{s}_{1}\left(k_{x} \kappa\right)\right]\left[(2 b-\tau) \cos \left(k_{y} \tau\right)+\tau \mathrm{s}_{1}\left(k_{y} \tau\right)\right] \\
{\left[\exp \left(-j k \sqrt{\kappa^{2}+\tau^{2}}\right) /\left(k \sqrt{\kappa^{2}+\tau^{2}}\right)\right] d \kappa d \tau /(2 \pi a b)}
\end{gathered}
$$

where

$$
\mathrm{s}_{1}(x)=\left\{\begin{array}{ll}
1 & \text { if } x=0 \\
\sin (x) / x & \text { if } x \neq 0
\end{array} .\right.
$$

Note that $s_{1}(x)$ is the un-normalized sinc function used in mathematics. Signal processing uses the normalized sinc function which is equal to $s_{1}(\pi x)$.

Define

$$
\begin{gathered}
k_{b}=\left|\mathbf{k}_{b}\right|=\sqrt{k_{x}^{2}+k_{y}^{2}} \\
\alpha=k_{x} / k \\
\beta=k_{y} / k \\
\mu=k_{b} / k=\sqrt{\alpha^{2}+\beta^{2}}
\end{gathered}
$$

Equation (16) can be written as [13]

$$
\begin{aligned}
z= & j k^{2} \int_{0}^{2 a} \int_{0}^{2 b}\left\{2 a \cos (\alpha k \kappa)-(\alpha / k) \frac{\partial}{\partial \alpha}[\sin (\alpha k \kappa) / \alpha]\right\} \\
& \left\{2 b \cos (\beta k \tau)-(\beta / k) \frac{\partial}{\partial \beta}[\sin (\beta k \tau) / \beta]\right\}\left[\exp \left(-j k \sqrt{\kappa^{2}+\tau^{2}}\right) /\left(k \sqrt{\kappa^{2}+\tau^{2}}\right)\right] d \kappa d \tau /(2 \pi a b)
\end{aligned}
$$




\section{The travelling plane wave case}

The double integrals in equations (15) and (22) can be converted to more complicated single integrals by converting to polar coordinates. Rhazi and Atalla [15] have given the result for the real part of equation (15) and indicated how to apply the method to obtain the imaginary part of equation (15). Rhazi and Atalla successfully used "a Gauss numerical integration scheme" written in FORTRAN to calculate the real part. However, when the authors of this paper evaluated their equations for the real part using the standard adaptive integral routine in MATLAB with its default settings, the adaptive integral routine did not converge when $k_{b}=k$. It was also discovered that it was faster to evaluate the real part of double integral in equation (15) using the standard adaptive double integral routine in MATLAB with its default settings when $k a$ and $k b$ were small. The reason is that Rhazi and Atalla's equations have terms which have singularities when $k_{b}=k$. Although these singularities do cancel out, they are sufficient to cause problems for MATLAB's adaptive integral routines. The authors have overcome this problem by writing the equations in a different form and also derived the modified equations for the imaginary part.

$$
\begin{gathered}
\mathrm{s}_{2}(x)=\mathrm{s}_{1}^{2}(x) \\
\mathrm{s}_{3}(x)=x \mathrm{~s}_{2}(x) \\
\mathrm{s}_{4}(x)= \begin{cases}0 & \text { if } x=0 \\
{\left[\mathrm{~s}_{1}(x)-\cos (x)\right] / x \text { if } x \neq 0}\end{cases} \\
\mathrm{s}_{5}(x)= \begin{cases}1 / 3 & \text { if } x=0 \\
\mathrm{~s}_{4}(x) / x \text { if } x \neq 0\end{cases} \\
s_{6}(x)=s_{1}(x)-s_{2}(x / 2) / 2 \\
s_{7}(x)= \begin{cases}0 & \text { if } x=0 \\
{\left[\mathrm{~s}_{2}(x / 2)-\mathrm{s}_{1}(x)\right] / x \text { if } x \neq 0}\end{cases} \\
s_{8}(x)=\mathrm{s}_{4}(x)-\mathrm{s}_{7}(x)
\end{gathered}
$$




$$
\begin{gathered}
T_{r 3}=k R \sin (\psi)\left\{\sum_{t=1}^{4}\left[-\mathrm{s}_{4}\left(a_{t} R\right)\right]\right\} /(2 b) \\
T_{i 3}=k R \sin (\psi)\left\{\sum_{t=1}^{4}\left[-\mathrm{s}_{6}\left(a_{t} R\right)\right]\right\} /(2 b) \\
T_{r 4}=k R^{2} \sin (\psi) \cos (\psi)\left[\sum_{t=1}^{4} \mathrm{~s}_{8}\left(a_{t} R\right)\right] /(4 a b) \\
T_{i 4}=k R^{2} \sin (\psi) \cos (\psi)\left[\sum_{t=1}^{4} \mathrm{~s}_{9}\left(a_{t} R\right)\right] /(4 a b)
\end{gathered}
$$

For $p$ equals $r$ or $i$

$$
\begin{gathered}
T_{p}=R\left(\sum_{t=1}^{4} T_{p t}\right) / 2, \\
z_{p}=\int_{0}^{\pi / 2} T_{p} d \psi / \pi .
\end{gathered}
$$

The value of equation (15) is

$$
z=z_{r}+j z_{i}
$$

\section{The simply supported mode case}

Leppington et. al. [13] give the results of converting the real part of equations (16) and (22) to a single integral by converting to polar co-ordinates. However their individual terms have singularities which cancel out. These singularities would cause difficulties with MATLAB's adaptive integral routines. Leppington et. al. also extend the range of integration from 0 to $\pi / 2$ radians to 0 to $2 \pi$ radians. This is undesirable from a numerical point of view. Thus the authors have rewritten the equations of Leppington et. al. without singularities in a format that is suitable for evaluation by MATLAB's adaptive integral routines. They have also derived the equations for the imaginary part which was not done by Leppington et. al..

The integrands $T_{r}$ and $T_{i}$ are first evaluated when both $\alpha$ and $\beta$ are non-zero.

$$
\begin{gathered}
T_{r 1}=k \sum_{t=1}^{4} \mathrm{~s}_{3}\left(a_{t} R / 2\right) \\
T_{i 1}=k \sum_{t=1}^{4} \mathrm{~s}_{1}\left(a_{t} R\right) \\
T_{r 21}=k R \cos (\psi) \sum_{t=1}^{4}\left[-\mathrm{s}_{4}\left(a_{t} R\right)\right] \\
T_{i 21}=k R \cos (\psi) \sum_{t=1}^{4}\left[-\mathrm{s}_{6}\left(a_{t} R\right)\right] \\
T_{r 22}=\left\{\sum_{t=1}^{4}\left[(-1)^{t} \mathrm{~s}_{1}\left(a_{t} R\right)\right]\right\} / \alpha \\
T_{i 22}=\left\{\sum_{t=1}^{4}\left[-(-1)^{t} \mathrm{~s}_{3}\left(a_{t} R / 2\right)\right]\right\} / \alpha
\end{gathered}
$$

For $p$ equals $r$ or $i$

$$
\begin{aligned}
T_{p 2} & =\left(\sum_{t=1}^{2} T_{p 2 t}\right) /(2 a) . \\
b_{t} & = \begin{cases}-1 & \text { if } t=1 \text { or } 4 \\
1 & \text { if } t=2 \text { or } 3\end{cases}
\end{aligned}
$$




$$
\begin{gathered}
T_{r 31}=k R \sin (\psi) \sum_{t=1}^{4}\left[-\mathrm{s}_{4}\left(a_{t} R\right)\right] \\
T_{i 31}=k R \sin (\psi) \sum_{t=1}^{4}\left[-\mathrm{s}_{6}\left(a_{t} R\right)\right] \\
T_{r 32}=\left\{\sum_{t=1}^{4}\left[b_{t} \mathrm{~s}_{1}\left(a_{t} R\right)\right]\right\} / \beta \\
T_{i 32}=\left\{\sum_{t=1}^{4}\left[-b_{t} \mathrm{~s}_{3}\left(a_{t} R / 2\right)\right]\right\} / \beta
\end{gathered}
$$

For $p$ equals $r$ or $i$

$$
\begin{gathered}
T_{p 3}=\left(\sum_{t=1}^{2} T_{p 3 t}\right) /(2 b) . \\
T_{r 41}=k R^{2} \sin (\psi) \cos (\psi) \sum_{t=1}^{4} \mathrm{~s}_{8}\left(a_{t} R\right) \\
T_{i 41}=k R^{2} \sin (\psi) \cos (\psi) \sum_{t=1}^{4} \mathrm{~s}_{9}\left(a_{t} R\right) \\
T_{r 42}=R \cos (\psi)\left\{\sum_{t=1}^{4}\left[-b_{t} \mathrm{~s}_{6}\left(a_{t} R\right)\right]\right\} / \beta \\
T_{i 42}=R \cos (\psi)\left\{\sum_{t=1}^{4}\left[b_{t} \mathrm{~s}_{4}\left(a_{t} R\right)\right]\right\} / \beta \\
T_{r 43}=R \sin (\psi)\left\{\sum_{t=1}^{4}\left[-(-1)^{t} \mathrm{~s}_{6}\left(a_{t} R\right)\right]\right\} / \alpha \\
T_{i 43}=R \sin (\psi)\left\{\sum_{t=1}^{4}\left[(-1)^{t} \mathrm{~s}_{4}\left(a_{t} R\right)\right]\right\} / \alpha \\
c_{t}=\{-1 \text { if } t=1 \text { or } 2 \\
T_{r 44}=\left\{\sum_{t=1}^{4}\left[c_{t} \mathrm{~s}_{3}\left(a_{t} R / 2\right)\right]\right\} /(k \alpha \beta) \\
T_{i 44}=\left\{\sum_{t=1}^{4}\left[c_{t} \mathrm{~s}_{1}\left(a_{t} R\right)\right]\right\} /(k \alpha \beta)
\end{gathered}
$$

For $p$ equals $r$ or $i$

$$
\begin{gathered}
T_{p 4}=\left(\sum_{t=1}^{4} T_{p 4 t}\right) /(4 a b), \\
T_{p}=R\left(\sum_{t=1}^{4} T_{p t}\right) / 2 .
\end{gathered}
$$

If $\alpha$ is zero and $\beta$ is non-zero, the integrands $T_{r}$ and $T_{i}$ are evaluated as follows. First $a_{1}$ and $a_{2}$ are redefined.

$$
\begin{aligned}
& a_{1}=k[1+\beta \sin (\psi)] \\
& a_{2}=k[1-\beta \sin (\psi)] \\
& T_{r 1}=k \sum_{t=1}^{2} s_{3}\left(a_{t} R / 2\right)
\end{aligned}
$$




$$
\begin{gathered}
T_{i 1}=k \sum_{t=1}^{2} s_{1}\left(a_{t} R\right) \\
T_{r 21}=R k \sin (\psi) \sum_{t=1}^{2}\left[-s_{4}\left(a_{t} R\right)\right] \\
T_{i 21}=R k \sin (\psi) \sum_{t=1}^{2}\left[-\mathrm{s}_{6}\left(a_{t} R\right)\right] \\
T_{r 22}=\left\{\sum_{t=1}^{2}\left[(-1)^{t} \mathrm{~s}_{1}\left(a_{t} R\right)\right]\right\} / \beta \\
T_{i 22}=\left\{\sum_{t=1}^{2}\left[-(-1)^{t} \mathrm{~s}_{3}\left(a_{t} R / 2\right)\right]\right\} / \beta
\end{gathered}
$$

For $p$ equals $r$ or $i$

$$
\begin{gathered}
T_{p 2}=\left(\sum_{t=1}^{2} T_{p 2 t}\right) /(2 b), \\
T_{p}=R \sum_{t=1}^{2} T_{p t} .
\end{gathered}
$$

If $\beta$ is zero and $\alpha$ is non-zero, the integrands $T_{r}$ and $T_{i}$ are evaluated as follows. First $a_{1}$ and $a_{2}$ are redefined.

$$
\begin{gathered}
a_{1}=k[1+\alpha \cos (\psi)] \\
a_{2}=k[1-\alpha \cos (\psi)] \\
T_{r 1}=k \sum_{t=1}^{2} s_{3}\left(a_{t} R / 2\right) \\
T_{i 1}=k \sum_{t=1}^{2} s_{1}\left(a_{t} R\right) \\
T_{r 21}=R k \cos (\psi) \sum_{t=1}^{2}\left[-s_{4}\left(a_{t} R\right)\right] \\
T_{i 21}=R k \cos (\psi) \sum_{t=1}^{2}\left[-\mathrm{s}_{6}\left(a_{t} R\right)\right] \\
T_{r 22}=\left\{\sum_{t=1}^{2}\left[(-1)^{t} \mathrm{~s}_{1}\left(a_{t} R\right)\right]\right\} / \alpha \\
T_{i 22}=\left\{\sum_{t=1}^{2}\left[-(-1)^{t} \mathrm{~s}_{3}\left(a_{t} R / 2\right)\right]\right\} / \alpha
\end{gathered}
$$

For $p$ equals $r$ or $i$

$$
\begin{gathered}
T_{p 2}=\left(\sum_{t=1}^{2} T_{p 2 t}\right) /(2 a), \\
T_{p}=R \sum_{t=1}^{2} T_{p t} .
\end{gathered}
$$

If $\alpha$ and $\beta$ are both zero, the integrands $T_{r}$ and $T_{i}$ are evaluated as follows.

$$
\begin{gathered}
T_{r}=2[1-\cos (k R)] \\
T_{i}=2 \sin (k R)
\end{gathered}
$$

Now the real part $z_{r}$ and the imaginary part $z_{i}$ of equations (16) and (22) can be calculated with a single numerical integration. 


$$
z_{p}=\int_{0}^{\pi / 2} T_{p} d \psi / \pi
$$

When numerically evaluating the integral in equation (94) with MATLAB's standard adaptive integral routine with its standard settings, it was found that it was necessary to assume that $\alpha$ and/or $\beta$ were zero if their magnitudes were less than $1 \times 10^{-6}$. The value of equations (16) and (22) is

$$
z=z_{r}+j z_{i}
$$

\section{The azimuthal average}

The azimuthal angle $\phi$ to the $x$-axis can be calculated.

$$
\phi=\arctan (\beta / \alpha)=\arctan \left(k_{y} / k_{x}\right)
$$

Then

$$
\begin{aligned}
& \alpha(\phi)=\mu(\phi) \cos (\phi), \\
& \beta(\phi)=\mu(\phi) \sin (\phi) .
\end{aligned}
$$

where $\mu(\phi)$ has been shown as a function of the azimuthal angle $\phi$ because it will sometimes depend on the direction of propagation, as is the case for a freely propagating wave on an orthotropic panel. The weighted average $z_{a v}$ of the impedance given by equations (47) or (94) over the azimuthal angle $\phi$ with weighting function $w(\phi)$ is

$$
z_{a z}=\int_{0}^{2 \pi} w(\phi) z(\phi) d \phi / \int_{0}^{2 \pi} w(\phi) d \phi .
$$

If $w(\phi)$ and $\mu(\phi)$ are symmetrical functions about the $x$ and $y$ axes, the ranges of integration over the azimuthal angle $\phi$ can be reduced to 0 to $\pi / 2$ radians by symmetry. If $w(\phi)$ and $\mu(\phi)$ are constant functions of the azimuthal angle $\phi$ and the rectangle $S$ is a square, the ranges of integration over the azimuthal angle $\phi$ can be reduced to 0 to $\pi / 4$ radians by symmetry. The weighting function $w(\phi)$ can be used to account for the fact that the wave impedance of an orthotropic panel varies with the azimuthal angle $\phi$ of propagation. The quantity $z_{a v}$ is what Leppington et al.'s [13] and Maidanik's [18, 19] approximate equations and the authors' [10-12, 20] previous approximate equations for the radiation efficiency of a rectangular panel in an infinite baffle are trying to approximate.

\section{The diffuse field incident average radiation impedance}

If the transverse velocity wave in the surface $S$ is forced by a plane sound wave with a wave number of $k_{i}$ incident from either side of the panel with an incidence angle of $\theta$ to the normal of the surface $S$ and an azimuthal angle of $\phi$ to the $x$-axis, then

$$
\mu(\phi)=\left(k_{i} / k\right) \sin (\theta)
$$

is constant as a function of the azimuthal angle $\phi$,

$$
\alpha(\phi)=\left(k_{i} / k\right) \sin (\theta) \cos (\phi)
$$

and

$$
\beta(\phi)=\left(k_{i} / k\right) \sin (\theta) \sin (\phi) .
$$

The incident wave number $k_{i}$ on the incident side may be different from the wave number $k$ on the radiating side if the incident and radiating sides are different and if the fluid media on each side of the panel are different. This will not normally be the case and $k_{i} / k$ will normally be equal to one. The travelling wave case should be used to calculate the radiation impedance for a transverse wave which is forced by an incident plane sound wave because the transverse waves reflected at 
the edges of the panel will have the freely propagating wave number of the panel rather than the forced wave number given by equations (100) and (21). Thus the anechoic edge conditions are approximate for the forced wave because there are no forced reflected waves. The radiation impedance of the freely propagating resonant reflected waves will have to be calculated separately using the appropriate boundary conditions.

The weighted incident diffuse sound field forced radiation impedance is the average of $z$ over all solid angles of incidence with weighting function $v(\theta, \phi)$ as shown in the following:

$$
\langle z\rangle=\int_{0}^{2 \pi} \int_{0}^{\pi / 2} v(\theta, \phi) z \sin (\theta) d \theta d \phi / \int_{0}^{2 \pi} \int_{0}^{\pi / 2} v(\theta, \phi) \sin (\theta) d \theta d \phi .
$$

If the weighting function $v(\theta, \phi)$ can be written as

$$
v(\theta, \phi)=p(\theta) w(\phi)
$$

then

$$
\langle z\rangle=\int_{0}^{\pi / 2} p(\theta) z_{a \nu} \sin (\theta) d \theta / \int_{0}^{\pi / 2} p(\theta) \sin (\theta) d \theta .
$$

\section{Simply supported mode}

If the isotropic rectangular panel $S$ is simply supported in the infinite rigid baffle, each of its transverse velocity modes has the transverse velocity amplitude on the surface of the panel given by equation (11). Each of these modes has $m$ and $n$ positive integer half wavelengths in the directions of the $x$ and $y$ axes respectively. Each mode is freely vibrating at its natural frequency or being forced to vibrate at a frequency which corresponds to a wave number of $k$ in the fluid medium on one side of the panel into which the panel is radiating sound. In this case the variables $\alpha$ and $\beta$ can only take the following discrete values.

$$
\begin{gathered}
\alpha=m \pi /(2 k a) \\
\beta=n \pi /(2 k b)
\end{gathered}
$$

However for the purposes of calculating the azimuthally averaged impedance, it is convenient to regard them as continuous variables. Because the variables $\alpha$ and $\beta$ are positive, azimuthal averaging only needs to be conducted over the range from 0 to $\pi / 2$.

The minimum value of $\mu$ in this case is

$$
\min (\mu)=\pi \sqrt{1 / a^{2}+1 / b^{2}} /(2 k) .
$$

For a square where $a=b=e$, the minimum is

$$
\min (\mu)=\pi /(\sqrt{2} k e) .
$$

Thus, it does not really make sense to calculate the impedance for the simply supported standing wave case when $\mu$ is less than the minimum value given by equation (108) or (109).

If the transverse velocity of the panel is due to freely propagating bending waves, then

$$
\mu=k_{b} / k=\sqrt{\omega_{c} / \omega}
$$

where $\omega_{c}$ is the angular critical frequency of the panel. The angular critical frequency is the angular frequency at which the wave number of the freely propagating bending waves in the panel equals the wave number of the sound in the surrounding compressible medium into which the panel is radiating sound. If the value of $\mu$ calculated using equation (110) is less than the minimum value of $\mu$ calculated using equation (108) or (109), then the radiation impedance for 
the simply supported mode case should be calculated using the minimum value of $\mu$ calculated using equation (108) or (109) rather than the value of $\mu$ calculated using equation (110).

\section{Approximate formulae}

Calculate

$$
\mu=\frac{k_{b}}{k}=\sin (\theta)
$$

where the second equality only applies if $k_{b} \leq k . \mu$ is the ratio of $k_{b}$ and $k$, which are respectively the transverse wave number in the rectangular panel and sound wave number in the surrounding compressible fluid medium into which the panel is radiating sound. $\theta$ is the angle of incidence of an incoming three dimensional plane wave in the surrounding fluid medium which could generate the transverse wave in the rectangular panel.

Calculate

$$
k e=2 k a b /(a+b),
$$

where $2 a$ and $2 b$ are the lengths of the sides of the rectangle and $2 e$ is the length of the sides of a equivalent square.

\section{$9.1 \mu \leq 1$}

If $\mu \leq 1$, calculate

$$
g=\sqrt{1-\mu^{2}}=\cos (\theta)
$$

and

$$
p=\sqrt{\pi /(2 k e)} .
$$

Set $w_{t r}=1.29, w_{t i}=0.9, w_{s r}=1.3$, and $w_{s i}=1.77$ and calculate $g_{x y}$ where $x$ equals $t$ for the travelling wave case or $s$ for the simply supported mode case and $y$ equals $r$ or $i$.

$$
g_{x y}=\min \left(w_{x y} p, 1\right) \text { where } x \text { equals } t \text { or } s \text { and } y \text { equals } r \text { or } i \text {. }
$$

Set $\alpha_{t}=2$ and $\alpha_{s}=8$. Calculate

$$
z_{x l r}=\alpha_{x} k^{2} a b / \pi
$$

If $g_{x r} \leq g \leq 1$, where $x$ equals $t$ or $s$, calculate

$$
z_{x h r}=1 / g .
$$

Set $\beta_{t r}=-0.07, \beta_{t i}=0.07, \beta_{s r}=0.4$ and $\beta_{s i}=-0.15$. If $g=0$ calculate

$$
z_{x h y}=z_{x 0 h y}=\max \left\{0.9616[2 /(3 p)]+\beta_{x y}, 0.001\right\} \text {. }
$$

If $0<g<g_{x r}$ calculate $z_{x 0 h r}$ using equation (118) and $z_{x m h r}$ using equation (117) with $g=g_{x r}$ for $x$ equals both $t$ and $s$. Interpolate in the inverse impedance domain as a function of $g$.

$$
z_{x h r}=g_{x r} /\left[\left(g_{x r}-g\right) / z_{x 0 h r}+g / z_{x m h r}\right] \text {. }
$$

Set $n_{t}=2$ and $n_{s}=3$. Calculate

$$
z_{x r}=1 / \sqrt[n_{x}]{1 / z_{x l r}^{n_{x}}+1 / z_{x h r}^{n_{x}}}
$$

where $z_{x l r}$ is calculated using equation (116) and $z_{x h r}$ is calculated using equations (117), (118), or (119). 
Calculate

$$
z_{t l i}=2 k[b H(a / b)+a H(b / a)] / \pi,
$$

where

$$
H(q)=\ln \left(\sqrt{1+q^{2}}+q\right)-\left(\sqrt{1+q^{2}}-1\right) /(3 q)
$$

Calculate

$$
z_{s l i}=2 k\left\{2 a \ln \left[\sqrt{1+(b / a)^{2}}+b / a\right]-2 b \ln \left[\sqrt{1+(a / b)^{2}}-a / b\right]\right\} / \pi
$$

If $g_{x i} \leq g \leq 1$, calculate

$$
\begin{gathered}
z_{t h i}=2 /\left(\pi k e g^{3}\right), \\
z_{s h i 1}=0.8 /(k e), \\
z_{s h i 2}=\max \left[2 \mu^{3} /\left(\pi k e g^{3}\right), 0.000001\right],
\end{gathered}
$$

and

$$
z_{s h i}=\left\{\begin{array}{ll}
z_{s h i 1} & \text { if } k e<1.2 \\
z_{s h i 2} & \text { if } k e \geq 1.2
\end{array} .\right.
$$

Set $n_{t}=4$ and $n_{s}=2$. Calculate

$$
z_{x i}=1 / \sqrt[n_{x}]{1 / z_{x l i}^{n_{x}}+1 / z_{x h i}^{n_{x}}} .
$$

using equations (118), (124) and (127).

If $g=0$, calculate

$$
\begin{gathered}
z_{t i}=z_{t 0 i}=\min \left(z_{t l i}, z_{t 0 h i}\right), \\
z_{s i 1}=1 / \sqrt{1 / z_{s l i}^{2}+1 / z_{s h i 1}^{2}},
\end{gathered}
$$

and

$$
z_{s i}=z_{s 0 i}=\max \left(z_{s i 1}, z_{s 0 h i}\right)
$$

using equations (118), (121) and (123).

If $0<g<g_{x i}$, calculate $z_{x 0 i}$ using equations (129) and (131), and $z_{x m i}$ using equation (128) with $g=g_{x i}$. Interpolate in the impedance domain as a function of $g$.

$$
z_{x i}=\left[\left(g_{x i}-g\right) z_{x 0 i}+g z_{x m i}\right] / g_{x i} .
$$

If $\mu \leq 1$, calculate

$$
z_{x}=z_{x r}+j z_{x i}
$$

where $z_{x r}$ is given by equation (120) and $z_{x i}$ is given by equations (128), (129), (131), or (132).

\section{$9.2 \mu>1$}

Else if $\mu>1$, set $h_{t r}=1.7, h_{t i}=1.3 h_{s r}=1.8$ and $h_{s i}=1.5$ and calculate $\mu_{x y}$ where $x$ equals $t$ or $s$ and $y$ equals $r$ and $i$. 


$$
\mu_{x y}=\sqrt{1+\pi h_{x y}^{2} /(2 k e)} \text { where } x \text { equals } t \text { or } s \text { and } y \text { equals } r \text { or } i \text {. }
$$

If $\mu \geq \mu_{x r}$, calculate

$$
\begin{gathered}
z_{t r}=2 /\left[\pi k e\left(\mu^{2}-1\right)^{3 / 2}\right], \\
z_{s r}=z_{t r}+\ln [(\mu+1) /(\mu-1)] /\left(\pi k e \mu \sqrt{\mu^{2}-1}\right) .
\end{gathered}
$$

If $1<\mu<\mu_{x r}$ calculate the real part $z_{x m r}$ using equation (135) or (136) with $\mu=\mu_{x r}$. Calculate the real part $z_{x 1 r}$ as described for the $\mu \leq 1$ case with $\mu=1$ which implies $g=0$.

Interpolate in the impedance domain as a function of $\mu$.

$$
z_{x r}=\left[\left(\mu_{x r}-\mu\right) z_{x 1 r}+(\mu-1) z_{x m r}\right] /\left(\mu_{x r}-1\right) .
$$

The calculation of the imaginary part for the travelling wave case when $\mu>1$ depends on the value of $k e$. If $k e \leq \sqrt{2}$, calculate the imaginary part $z_{t 1 i}$ as described for the $\mu \leq 1$ case with $\mu=1$. Calculate

$$
z_{t m i}=1 / \sqrt{\mu^{2}-1}
$$

Calculate

$$
z_{t i}=1 / \sqrt[4]{1 / z_{t 1 i}^{4}+1 / z_{t m i}^{4}}
$$

Else if $k e>\sqrt{2}$ proceed as follows

If $\mu \geq \mu_{t i}$, calculate

$$
z_{t i}=1 / \sqrt{\mu^{2}-1}
$$

If $1<\mu<\mu_{t i}$ calculate the imaginary part $z_{t m i}$ using Eq. (140) with $\mu=\mu_{t i}$.

Calculate the imaginary part $z_{t 1 i}$ as described for the $\mu \leq 1$ case with $\mu=1$ which implies $g=0$. Interpolate in the impedance domain as a function of $\mu$.

$$
z_{t i}=\left[\left(\mu_{t i}-\mu\right) z_{t 1 i}+(\mu-1) z_{t m i}\right] /\left(\mu_{t i}-1\right) .
$$

For the simply supported mode case, the imaginary part when $\mu>1$ is calculated as follows.

If $\mu \geq \mu_{s i}$, calculate

$$
z_{s i}=[1+1.3 /(\mu k e)] / \sqrt{\mu^{2}-1} .
$$

If $1<\mu<\mu_{s i}$ calculate the imaginary part $z_{s m i}$ using equation (142) with $\mu=\mu_{s i}$.

Calculate the imaginary part $z_{s 1 i}$ as described for the $\mu \leq 1$ case with $\mu=1$ which implies $g=0$. Interpolate in the impedance domain as a function of $\mu$.

$$
z_{s i}=\left[\left(\mu_{s i}-\mu\right) z_{s 1 i}+(\mu-1) z_{s m i}\right] /\left(\mu_{s i}-1\right) .
$$

If $\mu>1$, calculate

$$
z_{x}=z_{x r}+j z_{x i}
$$

where $z_{x r}$ is given by equations (135) or equations (137) and $z_{x i}$ is given by equations (139), (140), (141), (142) or(143). 
The azimuthally averaged one sided radiation impedance of a finite rectangular panel is given by equations (133) or (144).

\subsection{Incident diffuse sound field}

It is also possible to give approximate formulae for the real part of the normalized incident diffuse sound field forced radiation impedance in the travelling wave case if the weighting function is constant [20]. Using equation (115) define

$$
f=g_{t r}
$$

Using equation (116) define

$$
q=1 / z_{t l r} .
$$

Using equation (118) define

$$
h=1 / z_{t 0 h r} .
$$

Define

$$
\gamma=h / f-1 \text {. }
$$

Then the real part of the normalized incident diffuse sound field forced radiation impedance in the travelling wave case is

$$
\left\langle z_{t r}\right\rangle=\ln \left[\left(1+\sqrt{1+q^{2}}\right) /\left(f+\sqrt{f^{2}+q^{2}}\right)\right]+\ln \left[\left(h+\sqrt{h^{2}+q^{2}}\right) /\left(f+\sqrt{f^{2}+q^{2}}\right)\right] / \gamma .
$$

Note that this is also the incident diffuse sound field forced radiation efficiency of the panel.

\section{The effect of different wave types and boundary conditions}

For the travelling plane wave case, the normalized radiation wave impedance of an infinite panel is [17]

$$
z= \begin{cases}1 / \sqrt{1-\mu^{2}} & \text { if } 0 \leq \mu<1 \\ \infty & \text { if } \mu=1 \\ j / \sqrt{\mu^{2}-1} & \text { if } 1<\mu\end{cases}
$$

Thus for large values of $k e$, the real part of the impedance when $0 \leq \mu<1$ and the imaginary part of the impedance when $\mu>1$ are expected to be close to the values given by equation (150) and thus easier to approximate. The travelling plane wave case and the simply supported mode case are expected to have similar values of the real or the imaginary parts of their impedance in these two cases. Examination of equations (113), (117), (138), (140) and (142) shows that this is indeed the case. On the other hand, for large values of $k e$, the imaginary part of the impedance when $0 \leq \mu<1$ and the real part of the impedance when $\mu>1$ are expected to be close to the zero values given by equation (150) and thus harder to approximate. The travelling plane wave case and the simply supported mode case are expected to have different values of the imaginary or the real parts of their impedance in these two cases.

Examination of equation (116) shows that for small values of $k e$ and values of $\mu$ that are not too large, the simply supported mode case has a real part of its impedance that is four times or six decibels greater than the real part of the impedance for the travelling wave case. It should be noted that equation (116) can easily be derived as the limit for small values of $k e$ and $k_{b} e=\mu k e$ of the real parts of equations (15) and (16). Comparison of equation (135) and the term that is added to it in equation (136), show that they become equal for large values of $\mu$. This means that the real part of the impedance for the simply supported mode case tends to twice or 3 decibels greater than the real part of the impedance for the travelling wave case for large values of $k e$ and 
$\mu$. Comparison of equations (121), (122) and (123) shows that for small values of $k e$ and values of $\mu$ that are not too large, the simply supported mode case has an imaginary part of its impedance that is 2.37 times or $3.75 \mathrm{~dB}$ greater than the imaginary part of the impedance for the travelling wave case.

According to [21, 22], the real part of the radiation impedance of a clamped panel is twice as great as that of a simply supported panel for $\mu>1$ and ke large. More generally if the boundary conditions of the panel are zero displacement and a rotational stiffness, then zero rotational stiffness corresponds to the simply supported case and infinite rotational stiffness corresponds to clamped case. As the rotational stiffness varies from zero to infinity, the real part of the radiation impedance varies between the simply supported case and the clamped case and always lies in between these two cases.

Squicciarini et al. [23] have shown that real part of the normalized radiation impedance (radiation efficiency) of a point excited panel mounted in an infinite baffle depends on the edge conditions. In particular they have shown that when $\mu>1$ a freely supported panel has lower radiation efficiency than a simply supported panel and that a clamped panel has higher radiation efficiency than a simply supported panel except at very low values of $k_{b} e=\mu k e$ where the radiation is dominated by the fundamental drum mode.

\section{Origin of the approximate formulae}

The approximate formulae are based on the method used by the first author [20] to approximate the real part of the radiation impedance for the travelling wave case. The product of the airborne wave number and the half side length of the equivalent square (112) is calculated using equation (20) of Thomasson [8]. The expression for the side length of the equivalent square is also part of equation (7.7) of Leppington et al. [13] and the second term of equation (2.39a) of Maidanek [18]. Approximate formulae when ke $\square 1$ were derived for the $\mu=1$ case and for the $\mu \square 1$ case and later [11] for the $\mu \square 1$ case. Formulae for the upper limit $\mu$ (115) of applicability of the formula for the $\mu \square 1$ case and for the lower limit $\mu$ (134) of applicability of the formula for the $\mu \square 1$ case were derived. These formulae for the limits of applicability are based on the first author's research [20] and contain empirically derived constants. Interpolation in the inverse real impedance domain (119) and in the imaginary impedance domain (132) as a function of $g$ (113) was used for values of $\mu$ between the upper limit and $\mu=1$. Between $\mu=1$ and the lower limit, interpolation in the impedance domain (137), (141), (143) as a function of $\mu$ was used.

The values for the $k e \square 1$ case were combined [20] with the values for the $k e \square 1$ case by inverting the values, raising them to the power of $n$, summing these powers, taking the $n$th root and inverting the result (120), (128), (130), (139). There were some differences as to the order and manner in which this combination method and the interpolation method described above were applied. This was determined by comparison with the numerically calculated values and will not be discussed further here.

The combination method has been described above. Where did the equations that were combined come from? The equations (118) for the $\mu=1$ and $k e \square 1$ case are equation (B16) of Thomasson [8] with empirically determined correction terms. The equations (117) for the real part for the $\mu \square 1$ and $k e \square 1$ case are the first line of the infinite panel case given in equation (150). The equations (138), (140) and (142) for the imaginary part for the $\mu \square 1$ and $k e \square 1$ case are the third line of the infinite panel case given in equation (150). For the simply supported mode case, equation (142) also has an empirically determined correction term. The equations (135)and (136) for the real part when $\mu \square 1$ and $k e \square 1$ come from equation (7.7) of Leppington et al. [13]. The 
travelling wave case (135) only uses the second from equation (7.7) of Leppington et al. [13]. The imaginary part for the travelling wave case (124) when $\mu<1$ and $k e \square 1$ is based on a modification of the second term of equation (7.7) of Leppington et al. [13] for the case when $\mu<1$. The equation for the simply supported mode case (127) also includes an empirically determined $\mu^{3}$ factor. This equation has a different form (125) if $k e<1.2$.

For $k e \square 1$ and $\mu \leq 1$, the real part for the travelling wave case (116) is derived from the well known result for a rigid piston in an infinite baffle [20]. The simply supported version of equation of equation (116) is four times greater based on numerical and theoretical calculations. For ke $\square 1$ and $\mu \leq 1$, the imaginary part for the travelling wave case (121), (122) comes from equations (C10) and (C11) of Thomasson [8]. The simply supported case (123) was analytically calculated by the authors. Equations (145) to (149) for real part of the normalized incident diffuse sound field forced radiation impedance are from Davy [20].

\section{Accuracy of the approximate formulae}

In 2009, the first author [20] compared the approximate formulae for the real part of the azimuthally averaged normalized impedance (this is also the radiation efficiency) of a square panel mounted in an infinite rigid baffle for the travelling wave case when $0 \leq \mu \leq 1$ with numerical calculations made by Sato [24]. The comparisons were made for 15 values of $k e$ from 0.5 to 64 and for seven values of $\mu=\sin (\theta)$ from $\theta$ equals $0^{\circ}$ to $90^{\circ}$ in $15^{\circ}$ increments. The differences are shown in $\mathrm{dB}$ in table 1 of [20]. Although the constants in the approximate equations for this case have been slightly changed in this paper in order to be more consistent with the other approximate equations and as a result of having a wider range of values to compare against, the differences given in table 1 of [20] still give a good indication of the typical accuracy of the approximate equations in this case. The first author [20] also gave the mean, the standard deviation, the maximum and the minimum of the differences between Sato's [24] numerical calculations and the approximate equations given in [20] and the approximate equations given in four other publications. The first author [20] compared his own approximate equations and those of three other authors with Sato's [24] numerical calculations for the diffuse field excited case.

In 2014, because the first author's approximate equations only predicted the real part of the impedance for $\mu$ less than or equal to one, the authors [10] combined Thomasson's [8] high and low frequency equations, for $\mu$ less than or equal to one, for both the real and imaginary parts so that they covered the whole frequency range. These combined equations were compared with Thomasson's [8] tabulated numerical values for a square mounted in an infinite rigid baffle. The values of $k e$ ranged from 0.25 to 64 in half octave steps and the values of $\mu=\sin (\theta)$ from $\theta$ ranged from $0^{\circ}$ to $90^{\circ}$ in $15^{\circ}$ increments. There were also comparisons with five extra numerical values which were read from Thomasson's [8] graphs. The authors [10] also compared the first author's [20] approximate formulae for the real part of the impedance with Thomasson's [8] numerical calculations and discovered that they were a better approximation than the combined version of the real part of Thomasson's [8] formulae.

In [11], the authors extended their combined version of Thomasson's approximate formula to cover the case when $\mu$ was greater than one. These extended approximate formulae were compared with numerical calculations made by the authors for a square in an infinite rigid baffle for values of $k e$ ranging from 0.25 to 11.31 in half octave steps and for values of $\mu$ ranging from 1 to 10 in one tenth of a decade steps. The highest value of $k e$ was limited to 11.31 rather than 64 because of the increase in calculation time with increasing values of $k e \mu$ when numerically evaluating equation (15). 
Also in 2014, the authors [12] replaced their combined version of Thomasson's approximate formulae, for the case when $\mu$ is less than or equal to one, with the first author's [20] better performing approximate equations for the real part and developed new better approximate formulae for the imaginary part. Because the $\mu$ equals one values are used when interpolating to calculate some of the impedances when $\mu$ is greater than one, this change also changed some of the values when $\mu$ is greater than one. Comparisons between the approximate formulae and the numerically calculated impedances for a square panel in an infinite rigid baffle were made for the same values of variables as in [11]. In addition, because the authors' own numerically calculated values were used instead of Thomasson's tabulated values, comparisons were also made for the same $k e$ values when $\theta$ equals $70^{\circ}, 80^{\circ}$ and $85^{\circ}$.

In this paper, the reduction of one level of integration has enabled the authors to extend the range of $k e$ to be from 0.25 to 1024 in half octave steps while maintaining the same values of $\mu$ that were used in [12]. The one exception was the real part of the impedance when $\mu$ is greater than one. In this case the upper limit of $k e$ was only increased from 11.31 to 64 because of long calculation times even with the reduction of one level of integration.

All the authors' previous publications have only given comparisons between the approximate equations and the numerical calculations for the travelling wave case. In this paper the authors present approximate formulae for the simply supported mode case and also include calculations for a rectangle whose side lengths have ratio of 1:4 as well the calculations for a square. The introduction of a second case and the non-square rectangle and the significant extension of the range of ke means that the comparison tables of individual decibel differences would have quadrupled in number and increased more than a third in size. Thus in this paper, only the means, the standard deviations, the root mean square (rms) sums of the mean and standard deviation, the maxima and the minima of the differences are presented. Readers who are interested in the difference in impedance for individual values of the variables $k e$ and $\mu$ should consult the authors' previous papers on this topic to obtain a rough idea of the actual differences. The differences obtained with the versions of the formulae obtained in this paper are generally smaller. Some of these papers also give graphs of the numerically calculated values of the impedance.

For the simply supported wave case, the evaluation only occurs for values of $k e$ and $\mu$ which satisfy equation (108). For the simply supported mode case, some of the numerically calculated imaginary impedances for $\mu$ corresponding to values of $\theta$ less than $45^{\circ}$ were negative while the results produced by the approximate formulae were always positive. The decibel difference for these negative values could not be calculated unless the modulus of ratio was taken first. It was considered that taking modulus of the ratio would have been misleading. Thus in this one case all values for $\theta$ less than $45^{\circ}$ were excluded from the evaluation.

The results are shown in table 1 for a square and in table 2 for a rectangle whose side lengths have the ratio of 1:4. The most obvious thing is that, as speculated immediately after equation (150), the real part of the impedance when $0 \leq \mu<1$ and the imaginary part of the impedance when $\mu>1$ are more accurately predicted than the other cases. The agreement between the approximate formulae and the numerical calculations for the real part of the impedance when $\mu>1$ is good for large values of $k e$. The disagreement shown in table 1 for this case is due to ripple in the numerically calculated results as a function of $\mu$ for small values of $k e$. The agreement in the travelling wave case for the imaginary part when $\mu<1$ is also very good apart from some ripple in the numerically calculated results when $\mu$ is close to zero and $k e$ is of the order of two. For the imaginary part of the impedance for the simply supported mode case when $\mu<1$, it needs to be remembered that only differences when $\mu$ is greater 0.71 are analysed. This is because some 
of the numerically calculated values are negative when $\mu$ is less than 0.71 . In this region the impedance oscillates between positive and negative values. The agreement for the $\mu$ equals one case is good. This is slightly surprising since the infinite panel value is infinite in this case. Tables 1 and 2 also show the results for the real part of the impedance for the incident diffuse sound field forced travelling wave case. As would be expected, the results are similar to the real parts of the azimuthally averaged impedance when $\mu \leq 1$ for the travelling wave case and for the simply supported mode case. The magnitude of the statistics of the differences for the non-square rectanglar case in Table 2 are usually slightly greater than, but similar to, the results for the square case in Table 1.

Table 1. The mean, the standard deviation, the root mean square (rms) sum of the mean and standard deviation, the maxima and the minima of the differences in decibels between the approximate formulae and the numerical calculations for the real and imaginary parts of the azimuthally averaged radiation wave impedance of an isotropic square panel mounted in an infinite rigid baffle. The travelling wave case is denoted by TW and the simply supported wave case is denoted by SS. $\mu$ is the ratio of the wave number of the transverse vibrational wave in the panel to the wave number of sound in air. Also shown are the results for the real part of the incident diffuse sound field excited case.

\begin{tabular}{llllll}
\hline Case & Mean $(\mathrm{dB})$ & $\begin{array}{l}\text { Standard } \\
\text { Deviation } \\
(\mathrm{dB})\end{array}$ & $\begin{array}{l}\text { Root } \\
\text { Square }(\mathrm{dB})\end{array}$ & $\begin{array}{l}\text { Maximum } \\
(\mathrm{dB})\end{array}$ & $\begin{array}{l}\text { Minimum } \\
(\mathrm{dB})\end{array}$ \\
\hline Real TW $\mu \leq 1$ & 0.00 & 0.13 & 0.13 & 0.36 & -0.61 \\
\hline Real SS $\mu \leq 1$ & 0.01 & 0.14 & 0.14 & 0.35 & -0.58 \\
\hline Imag TW $\mu \leq 1$ & -0.02 & 0.41 & 0.41 & 2.11 & -1.56 \\
\hline Imag SS $\mu \leq 1$ & -0.25 & 0.73 & 0.77 & 2.73 & -1.38 \\
\hline Real TW $\mu \geq 1$ & -0.02 & 0.91 & 0.91 & 6.23 & -2.44 \\
\hline Real SS $\mu \geq 1$ & -0.67 & 1.22 & 1.39 & 3.21 & -5.25 \\
\hline Imag TW $\mu \geq 1$ & 0.01 & 0.09 & 0.09 & 0.42 & -0.35 \\
\hline Imag SS $\mu \geq 1$ & 0.02 & 0.09 & 0.09 & 0.35 & -0.49 \\
\hline Real Diffuse & 0.01 & 0.11 & 0.11 & 0.25 & -0.26 \\
\hline
\end{tabular}

Figures 1 and 2 show the maxima and the minima of the differences in decibels between the approximate formulae and the numerical calculations for the real and imaginary parts of the azimuthally averaged radiation wave impedance of an isotropic square panel mounted in an infinite rigid baffle as a function of $k e$. Figure 1 shows the maximum and minima across $\mu \leq 1$ while Figure 2 shows the maximum and minima across $\mu \geq 1$. Figure 1 also shows the differences for the real part of the incident diffuse sound field excited case. Figure 3 shows the maxima and the minima of the differences in decibels between the approximate formulae and the numerical calculations for the real and imaginary parts of the azimuthally averaged radiation wave impedance of an isotropic square panel mounted in an infinite rigid baffle across values of $k e$ as a function of $\mu$. As in the tables, for the simply supported wave case, the evaluation only occurs across values of $k e$ and $\mu$ which satisfy equation (108) and for the imaginary part in the simply supported case all values of $\mu$ less than 0.71 ( $\theta$ less than $45^{\circ}$ ) were excluded from the 
evaluation. The $\mu=0.1$ values in Figure 3 are actually $\mu=0$ values. This change was made so that the $\mu=0$ values could be included on the logarithmically scaled graph.

From Figure 2, it can be seen that the differences in the $\mu \geq 1$ case are very small for large values of $k e$. The differences for the imaginary part in the $\mu \geq 1$ case are small for all values of $k e$, while the differences for the real part in the $\mu \geq 1$ case are large for small values of $k e$, because of the oscillating behaviour of the numerically calculated impedance. Figure 1 shows that the roles are reversed in the $\mu \leq 1$ case. The differences of the real part in the $\mu \leq 1$ case are reasonably small for all values of $k e$, while the differences of the imaginary part in the $\mu \leq 1$ case are larger except for very small values of $k e$. The differences for the real part of the incident diffuse sound field excited case are also small for all values of ke. Figure 3 shows that the biggest differences occur for the real part of the impedance for large values of $\mu$. Figure 2 shows that this is due to large differences for small values of $k e$.

Table 2. The mean, the standard deviation, the root mean square (rms) sum of the mean and standard deviation, the maxima and the minima of the differences in decibels between the approximate formulae and the numerical calculations for the real and imaginary parts of the azimuthally averaged radiation wave impedance of a rectangular panel, whose side lengths are a ratio of four, mounted in an infinite rigid baffle. The travelling wave case is denoted by TW and the simply supported wave case is denoted by SS. $\mu$ is the ratio of the wave number of the transverse vibrational wave in the panel to the wave number of sound in air. Also shown are the results for the real part of the incident diffuse sound field excited case.

\begin{tabular}{llllll}
\hline Case & Mean $(\mathrm{dB})$ & $\begin{array}{l}\text { Standard } \\
\text { Deviation } \\
(\mathrm{dB})\end{array}$ & $\begin{array}{l}\text { Root } \\
\text { Square }(\mathrm{dB})\end{array}$ & $\begin{array}{l}\text { Maximum } \\
(\mathrm{dB})\end{array}$ & $\begin{array}{l}\text { Minimum } \\
(\mathrm{dB})\end{array}$ \\
\hline Real TW $\mu \leq 1$ & 0.10 & 0.35 & 0.36 & 1.24 & -0.43 \\
\hline Real SS $\mu \leq 1$ & -0.09 & 0.23 & 0.24 & 0.37 & -0.96 \\
\hline Imag TW $\mu \leq 1$ & -0.01 & 0.49 & 0.49 & 1.53 & -1.79 \\
\hline Imag SS $\mu \leq 1$ & -0.26 & 0.94 & 0.97 & 5.40 & -2.08 \\
\hline Real TW $\mu \geq 1$ & 0.18 & 1.06 & 1.08 & 5.46 & -2.33 \\
\hline Real SS $\mu \geq 1$ & -0.45 & 1.06 & 1.15 & 2.62 & -4.93 \\
\hline Imag TW $\mu \geq 1$ & 0.03 & 0.16 & 0.16 & 0.82 & -0.34 \\
\hline Imag SS $\mu \geq 1$ & 0.01 & 0.14 & 0.14 & 0.74 & -0.94 \\
\hline Real Diffuse & 0.11 & 0.35 & 0.37 & 1.02 & -0.27 \\
\hline
\end{tabular}




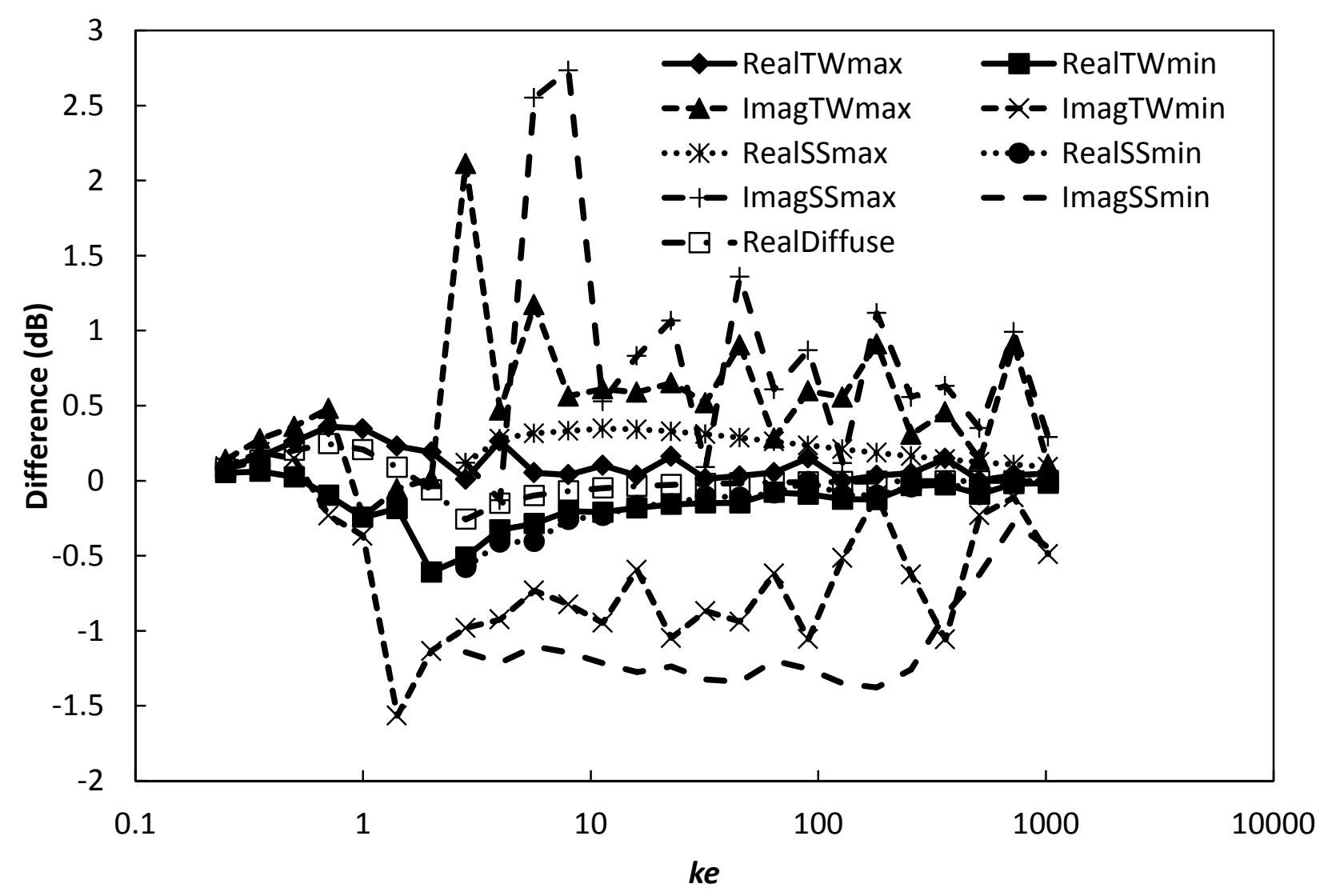

Figure 1. The maxima and the minima of the differences in decibels between the approximate formulae and the numerical calculations for the real and imaginary parts of the azimuthally averaged radiation wave impedance of an isotropic square panel mounted in an infinite rigid baffle across values of $\mu \leq 1$ as a function of $k e$. The travelling wave case is denoted by TW and the simply supported wave case is denoted by SS. $\mu$ is the ratio of the wave number of the transverse vibrational wave in the panel to the wave number of sound in air. Also shown are the differences for the real part of the incident diffuse sound field excited case. 


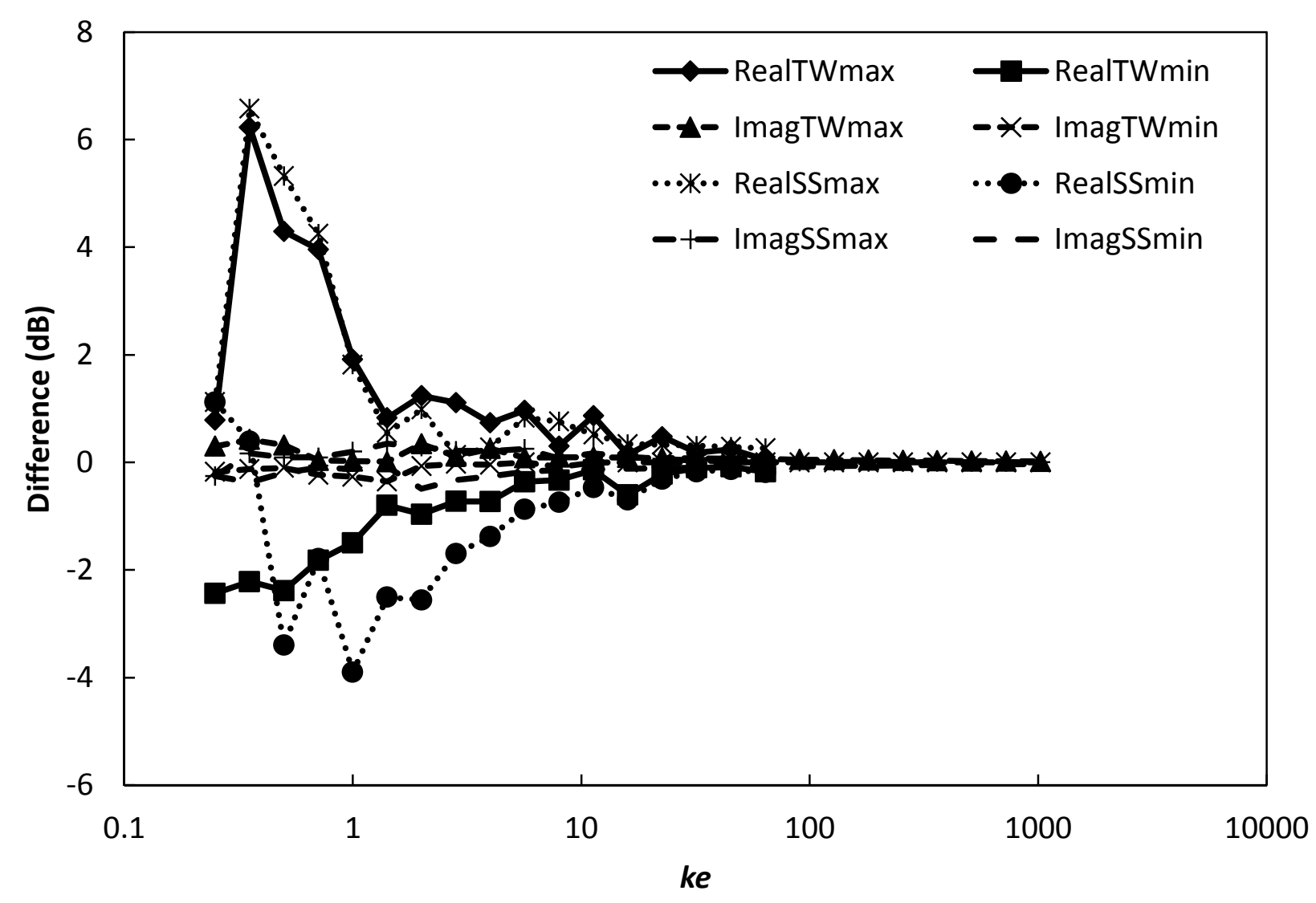

Figure 2. The maxima and the minima of the differences in decibels between the approximate formulae and the numerical calculations for the real and imaginary parts of the azimuthally averaged radiation wave impedance of an isotropic square panel mounted in an infinite rigid baffle across values of $\mu \geq 1$ as a function of $k e$. The travelling wave case is denoted by TW and the simply supported wave case is denoted by SS. $\mu$ is the ratio of the wave number of the transverse vibrational wave in the panel to the wave number of sound in air. 


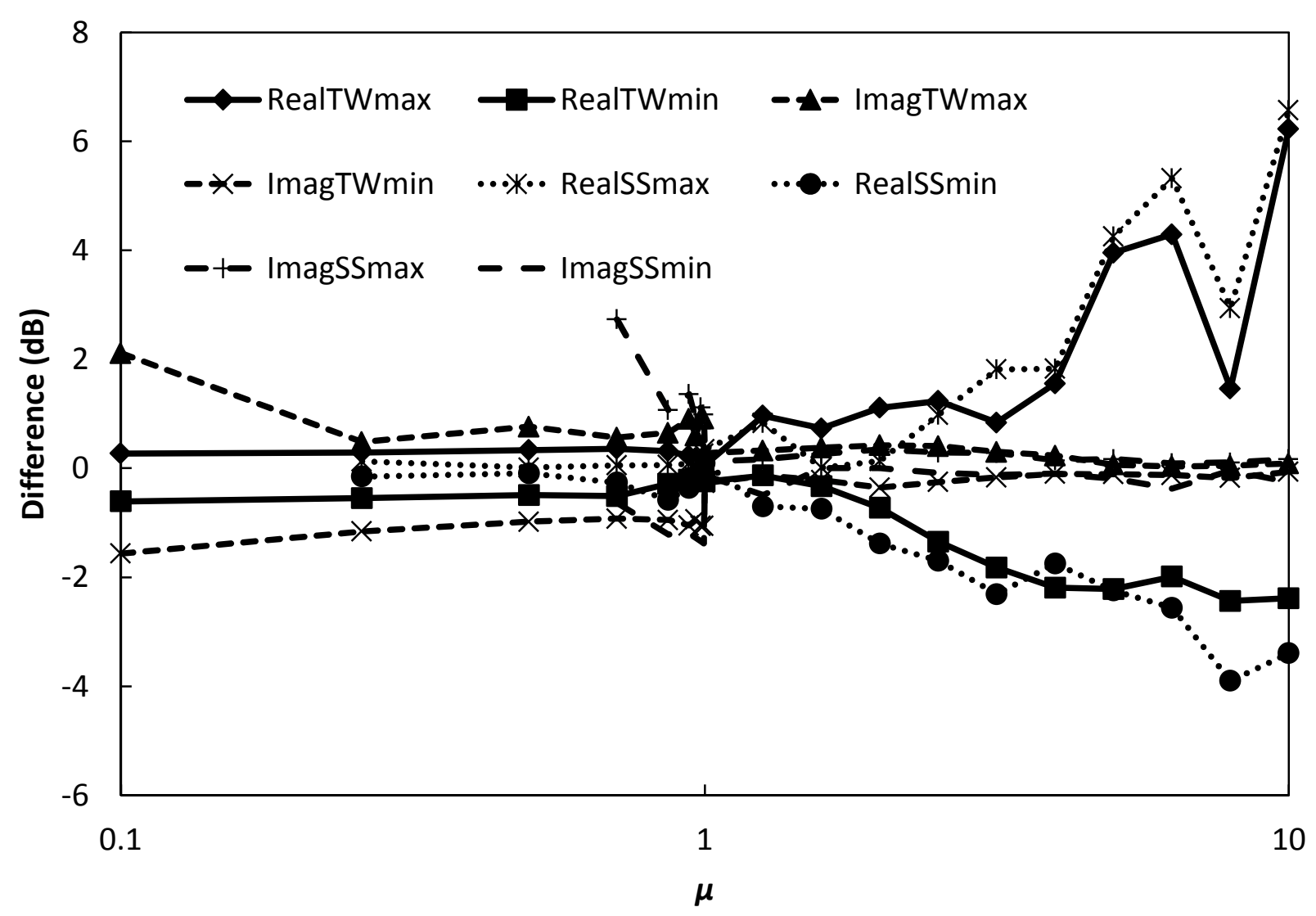

Figure 3. The maxima and the minima of the differences in decibels between the approximate formulae and the numerical calculations for the real and imaginary parts of the azimuthally averaged radiation wave impedance of an isotropic square panel mounted in an infinite rigid baffle across values of $\mu \geq 1$ as a function of $k e$. The travelling wave case is denoted by TW and the simply supported wave case is denoted by SS. $\mu$ is the ratio of the wave number of the transverse vibrational wave in the panel to the wave number of sound in air.

\section{Calculation of radiation efficiency}

One of the main uses of the formulae given in this paper is to calculate the radiation efficiency which is just the real part of the normalized radiation impedance. If a thin isotropic panel, mounted in an infinite rigid baffle is excited by an airborne incident diffuse sound field, then the transverse vibration of the panel consists of a forced non-resonant field and a freely propagating resonant field. The ratio $r$ of the resonant vibrational energy to the non-resonant vibrational energy level of a panel which has been excited by a diffuse sound field is [25]

$$
r=\pi \omega_{c} \sigma_{r} /(4 \omega \eta) \text {, }
$$

where $\eta$ is the total in situ damping loss factor of the panel. $\sigma_{r}$ is the radiation efficiency of the resonant transverse vibration field. This is calculated using the equations for the real part of the normalized radiation impedance for the simply supported case given in this paper with the value of $\mu$ given by equation (110) and limiting $\mu$ to be greater than or equal to the minimum value given by equation (108) or (109). $\sigma_{n r}$ is the radiation efficiency of the non-resonant vibration field. This is calculated using equation (105) with constant weighting functions or the approximate equation (149) for the real part of the normalized diffuse field incident radiation impedance for the travelling wave case. The radiation efficiency of the airborne diffuse sound field excited panel is the weighted average. 


$$
\sigma_{a}= \begin{cases}\left(r \sigma_{r}+\sigma_{n r}\right) /(r+1) & \text { if } \omega<\omega_{c} \\ \sigma_{r} & \text { if } \omega \geq \omega_{c}\end{cases}
$$

Above the angular critical frequency, it is not possible to distinguish between the resonant and the non-resonant transverse vibrational fields in the panel.

The variable $r$ is also the ratio of the power radiated by the resonant vibrational fields to the power radiated by the vibrational near fields for a panel excited by point forces acting at right angles to the panel [5]. The radiation efficiency of a panel excited by point forces acting at right angles to the panel is (see equation (28) of [26])

$$
\sigma_{p}=\left\{\begin{array}{ll}
\sigma_{r}(1+1 / r) & \text { if } \omega<\omega_{c} \\
\sigma_{r} & \text { if } \omega \geq \omega_{c}
\end{array} .\right.
$$

The ratio of the power radiated by the resonant vibrational fields to the power radiated by the vibrational near fields for a panel excited by line forces acting at right angles to the panel [5] is

$$
r_{l}=\sqrt{\omega_{c} / \omega} \sigma_{r} /(2 \eta) \text {. }
$$

The radiation efficiency of a panel excited by line forces acting at right angles to the panel is

$$
\sigma_{l}=\left\{\begin{array}{ll}
\sigma_{r}\left(1+1 / r_{l}\right) & \text { if } \omega<\omega_{c} \\
\sigma_{r} & \text { if } \omega \geq \omega_{c}
\end{array} .\right.
$$

Thus although the fundamental radiation efficiencies do not depend on the damping loss factor, the above equations show that the radiation efficiency of an excited panel does depend on the damping loss factor when $\omega<\omega_{c}$. This has also been shown in [23, 27].

\section{Conclusions}

This paper extends the previous definition of the radiation impedance used by the authors so that it covers standing waves as well as plane travelling waves. It gives single integral versions of the equations, for the normalized radiation impedance of a rectangular panel set in an infinite baffle, which remove the singularities so that the integrals can be successfully and effectively evaluated using adaptive integration. These equations are given for the real and imaginary parts for both the travelling plane wave case and for the simply supported mode case. These two cases have usually been treated separately in the literature. This paper gives a uniform treatment of both cases.

Approximate formulae for the azimuthally averaged normalized radiation impedance of a rectangular panel set in an infinite baffle are given for all the cases considered in this paper. An approximate formula for the real part of the normalized impedance for the incident diffuse sound field excited case is also given. Prior to the authors' research, most attempts to produce approximate formulae have not covered the whole range of variables.

These approximate formulae are compared with numerical calculations and reasonable agreement is obtained in most situations. The faster speed of the single integral version of the equations, compared to the previous double integral versions used previously by the authors, has enabled the comparisons to be made over a bigger range of variables.

The effect of different wave types and boundary conditions on the radiation impedance is discussed. Because at least two different types of transverse vibrational fields are usually established in a finite panel when it is excited, formulae are given for calculating the radiation impedance of the panel due to the combined effect of these two different types of fields when the panel is excited by an incident diffuse sound field or by transverse point or line forces. 


\section{References}

[1] J.F. Allard, N. Atalla, Propagation of sound in porous media : modelling sound absorbing materials, 2nd ed., John Wiley \& Sons, Ltd, Chichester, 2009.

[2] J. Brunskog, The forced sound transmission of finite single leaf walls using a variational technique, J. Acoust. Soc. Am., 132 (2012) 1482-1493.

[3] J.L. Davy, Predicting the sound insulation of single leaf walls - extension of Cremer's model, J. Acoust. Soc. Am., 126 (2009) 1871-1877.

[4] J.L. Davy, The improvement of a simple theoretical model for the prediction of the sound insulation of double leaf walls, J. Acoust. Soc. Am., 127 (2010) 841-849.

[5] J.L. Davy, Sound transmission of cavity walls due to structure borne transmission via point and line connections, J. Acoust. Soc. Am., 132 (2012) 814-821.

[6] C.-H. Jeong, Converting Sabine absorption coefficients to random incidence absorption coefficients, J. Acoust. Soc. Am., 133 (2013) 3951-3962.

[7] S.-I. Thomasson, On the absorption coefficient, Acust., 44 (1980) 265-273.

[8] S.-I. Thomasson, Theory and experiments on the sound absorption as function of the area, Report TRITA-TAK-8201, in, Department of Technical Acoustics, Royal Institute of Technology, Stockholm,Sweden, 1982, pp. 1-68.

[9] J.L. Davy, The directivity of the sound radiation from panels and openings, J. Acoust. Soc. Am., 125 (2009) 3795-3805.

[10] J.L. Davy, D.J. Larner, R.R. Wareing, J.R. Pearse, The specific forced radiation wave impedance of a finite rectangular panel excited by a plane sound wave, in: The 21st International Congress on Sound and Vibration, Beijing, China 2014, pp. 1-8.

[11] J.L. Davy, D.J. Larner, R.R. Wareing, J.R. Pearse, The average specific forced radiation wave impedance of a finite rectangular panel, J. Acoust. Soc. Am., 136 (2014) 525-536.

[12] J.L. Davy, D.J. Larner, R.R. Wareing, J.R. Pearse, Approximate formulae for the average one sided specific radiation wave impedance of a finite rectangular panel, in: Inter-noise 2014, Melbourne, Australia, , 2014, pp. 1-10.

[13] F.G. Leppington, E.G. Broadbent, K.H. Heron, The acoustic radiation efficiency of rectangular panels, Proc. R. Soc. London, Ser. A, 382 (1982) 245-271.

[14] W.L. Li, H.J. Gibeling, Determination of the mutual radiation resistances of a rectangular plate and their impact on the radiated sound power, J. Sound Vib., 229 (2000) 1213-1233.

[15] D. Rhazi, N. Atalla, A simple method to account for size effects in the transfer matrix method, J. Acoust. Soc. Am., 127 (2010) EL30-EL36.

[16] G. Xie, D.J. Thompson, C.J.C. Jones, The radiation efficiency of baffled plates and strips, J. Sound Vib., 280 (2005) 181-2009.

[17] F. Fahy, Sound and structural vibration - Radiation, transmission and response, Academic Press, London, 1985.

[18] G. Maidanik, Response of ribbed panels to reverberant acoustic fields, J. Acoust. Soc. Am., 34 (1962) 809-826.

[19] G. Maidanik, Erratum: Response of ribbed panels to reverberant acoustic fields, J. Acoust. Soc. Am., 57 (1975) 1552.

[20] J.L. Davy, The forced radiation efficiency of finite size flat panels that are excited by incident sound, J. Acoust. Soc. Am., 126 (2009) 694-702.

[21] F.G. Leppington, E.G. Broadbent, K.H. Heron, Acoustic radiation from rectangular panels with constrained edges, Proc. R. Soc. London, Ser. A, 393 (1984) 67-84.

[22] P.W. Smith, Coupling of sound and panel vibration below the critical frequency, J. Acoust. Soc. Am., 36 (1964) 1516-1520. 
[23] C. Squicciarini, D.J. Thompson, R. Corradi, The effect of different combinations of boundary conditions on the average radiation efficiency of rectangular plates, J. Sound Vib., 333 (2014) 3931-3948.

[24] H. Sato, On the mechanism of outdoor noise transmission through walls and windows - A modification of infinite wall theory with respect to radiation of transmitted wave, J. Acoust. Soc. Jpn., 29 (1973) 509-516.

[25] M.J. Crocker, A.J. Price, Sound transmission using statistical energy analysis, J. Sound Vib., 9 (1969) 469-486.

[26] J.L. Davy, J.P. Mahn, C. Guigou-Carter, M. Villot, The prediction of flanking sound transmission below the critical frequency, J. Acoust. Soc. Am., 132 (2012) 2359-2370.

[27] Y. Kou , B. Liu, J. Tian, Radiation efficiency of damped plates, J. Acoust. Soc. Am., 137 (2015) 1032-1035. 